\title{
Enhanced Sensitivity to Stress and Drug/Alcohol Craving in Abstinent Cocaine-Dependent Individuals Compared to Social Drinkers
}

\author{
Helen C Fox",', Kwang-lk A Hong', Kristen Siedlarz' and Rajita Sinha' \\ 'Department of Psychiatry, Yale University School of Medicine, Substance Abuse Center, Connecticut Mental Health Center, New Haven, \\ $C T$, USA
}

\begin{abstract}
Chronic exposure to cocaine is associated with neuroadaptions in stress and reward circuits that may increase susceptibility to relapse. We examined whether there are alterations in stress response and craving in abstinent cocaine-dependent individuals compared with a demographically matched group of non-addicted socially drinking community controls. Forty treatment-engaged abstinent cocaine patients (I7F/23M) and 40 controls (I 9F/2IM) were exposed to a brief 5 min guided imagery of individually calibrated stressful situations, personal drug/alcohol-related situation and a neutral-relaxing situation, one imagery per session, presented in random order. Craving, anxiety, emotion rating scales, and physiological measures were assessed. Cocaine patients reported significantly higher and more persistent stress- and cue-induced drug/alcohol craving, negative emotions, and physiological responses compared with social drinkers. In cocaine patients, stress- and cue-induced drug craving was accompanied by increased anger, fear, sadness, heart rate, and SBP. Controls reported minimal stress-induced craving and only increases in anxiety and SBP during stress exposure. Cue-induced alcohol craving was accompanied only by an increase in relaxed state. Females reported increased stress-induced anxiety and sadness compared with males, while males were emotionally and physiologically more reactive in the cue condition. These findings are the first to document functional alterations in stress- and reward-related affect and physiology in recently abstinent cocaine patients that is marked by an enhanced sensitivity to stress- and drug-related cue exposure. These data suggest that recovery from chronic cocaine abuse could be hampered by a hyper-responsive stress- and drug-craving state that increases cocaine relapse susceptibility.

Neuropsychopharmacology (2008) 33, 796-805; doi:I0.I038/sj.npp. I30 I470; published online I3 June 2007
\end{abstract}

Keywords: cocaine abuse; stress; drug cues; emotion; gender; craving

\section{INTRODUCTION}

Escalating patterns of cocaine self-administration in laboratory animals results in drug-induced neuroadaptions in the stress and reward circuits of the brain that are commonly associated with the transition to addiction (Weiss, 2005; Koob et al, 2004). For example, raised reward thresholds following drug exposure often provide an index for increased abstinence behaviors (Kenny and Markou, 2005; Schulteis et al, 1995; Kenny et al, 2006). In humans, these have been associated with the protracted withdrawal symptoms documented in early abstinence that include increased irritability, restlessness, depressed mood and anxiety, high cravings (Sinha, 2001; Kampman et al, 1998, 2001a) and altered stress responses, which have been associated with relapse in both alcoholics (Adinoff et al, 2005; Breese et al, 2005; Brady et al, 2006) and cocainedependent individuals (Sinha et al, 2006).

*Correspondence: Dr HC Fox, Department of Psychiatry, Yale University School of Medicine, Substance Abuse Center, Connecticut Mental Health Center, 34 Park Street, New Haven, CT 06519, USA, Tel: + I 203974 7262, E-mail: helen.fox@yale.edu

Received 12 December 2006; revised 26 February 2007; accepted 19 March 2007
The conceptualization of cocaine abuse as a chronic stressor increasing hedonic dysregulation (Koob and Le Moal, 1997, 2001) is growing in acceptance as a model of addiction; however, to date, there remains a paucity of data examining the nature of these mechanisms in humans. As stress- and drug cue-related stimuli have been associated with the activation of both stress and appetitive brain systems (Kalivas and Stewart, 1991; Kilts et al, 2004), the current study aims to investigate whether cocaine patients will demonstrate dysregulation of hedonic set-point by displaying enhanced negative distress and physiological response to stress- and cue-related craving compared with well-matched non-addicted socially drinking community controls.

Increased behavioral sensitivity following cocaine exposure and during abstinence have predominantly been documented within the animal literature. Findings have shown functional and structural changes in autonomic, reward, and stress-related brain regions as well as modulations of neural mechanisms associated with behavioral sensitivity. Following chronic cocaine selfadministration schedules, alterations to arousal-related dopaminergic and noradrenergic systems have been reported including increased densities of norepinephrine 
transporter binding in the hypothalamic nuclei and entorhinal cortex (Beveridge et al, 2005) as well as changes in the density of D1 and D2 receptors within striatal regions (Nader et al, 2002; Moore et al, 1998). Pre-exposure to cocaine in rats has also been associated with long-term sensitization of the corticotrophin-releasing factor (CRF) systems (Erb et al, 2003) and stress exposure has been shown to influence locomotor sensitization to cocaine (Lepsch et al, 2005; Haile et al, 2001).

Previous studies from our laboratory, which compared stress- and cue-related emotional and physiological response with that induced by a neutral imagery scenario, have served to highlight the possibility that enhanced negative emotion and physiological response may be representative of the pathophysiology of the craving state in recently abstinent cocaine patients. Increases in both stress- and cue-induced physiological output, HPA axis activation, and negative emotion were observed concomitant with increased cocaine craving (Sinha et al, 2000, 2003; Fox et al, 2005, 2006). However, no previous work has contrasted these findings to a group of well-matched noncocaine addicted control individuals. In the present study, social drinkers were used as controls owing to the fact that this group has demonstrated increased physiological reactivity and desire for alcohol following cue exposure (Cooney et al, 1987; Greeley et al, 1993), thereby allowing for a comparison of the craving state between a nonaddicted and addicted group of patients. Comparisons between both groups are necessary in order to fully determine (1) the extent to which a potential dysregulation of neural stress and reward circuits underlies stress- and cue-induced craving in abstinent cocaine patients and (2) to help clarify the emotional and physiological components associated with an increased sensitivity to stress- and cue-related cocaine craving.

A secondary aim of this study was to assess the impact of gender on these hedonic regulatory systems, as previous research has established that sex differences may impact on allostatic load as well as diseases associated with stressrelated dysfunction (Brunova, 2004; Pariante, 2003). Furthermore, recent findings have highlighted that some of the physiological and biochemical mechanisms associated with stress- and cue-induced craving may be gender sensitive. Sex differences have been reported in HPA drive, physiological response, and subjective reactivity to stress and drug cue in recently abstinent cocaine patients (Fox et al, 2006; Back et al, 2005). We therefore hypothesize that cocaine patients will demonstrate an enhanced drug/alcohol craving and emotional and physiological response to stressand cue-related imagery compared with social drinkers and that gender plays an important role in these effects.

\section{METHODS}

\section{Participants}

Forty treatment-seeking cocaine-dependent individuals (17F/23M) and 40 socially drinking controls (19F/21M) participated in the current study. All participants were recruited via advertisements placed either on-line or in local newspapers and magazines. Current dependence in the potential cocaine participants was determined using the
Structured Clinical Interview for the Diagnostic and Statistical Manual of Mental Disorders IV (SCID IV; First et al, 1997). They also tested for positive urine toxicology screens upon entry into the in-patient treatment and research facility at the Clinical Neuroscience Research Unit (CNRU) of the Connecticut Mental Health Center (CMHC). Exclusion criteria for cocaine-patients included DSM-IV dependence for any drug other than cocaine, alcohol, or nicotine. All controls were social drinkers (25 drinks or less per month) as classified by the Quantity Frequency Variability Index (Callahan and Cisin, 1968) and were excluded if they met current or lifetime dependence criteria for alcohol or any other illicit drug. Individuals who were on prescribed medications or were in need of treatment for any other psychiatric or medical conditions and failed to meet good health requirements were excluded. Participants underwent stringent medical assessments that included electrocardiography and laboratory tests of renal, hepatic, pancreatic, hematopoietic, and thyroid function to ensure good physical health. All participants gave written consent and the Human Investigation Committee of the Yale University School of Medicine approved the study.

\section{Procedures}

Cocaine participants were admitted to the CNRU of the Connecticut Mental Health Center (CMHC) for 2-4 weeks of in-patient treatment and study participation. The CNRU is a locked in-patient treatment research facility with no access to alcohol or drugs and very limited access to visitors. Drug testing was conducted regularly to ensure drug abstinence. The unit also allows four smoke breaks during which time patients are permitted to smoke 1-2 cigarettes per occasion. Socially drinking control participants were admitted to the in-patient research unit of the Yale General Clinical Research Center (GCRC) at Yale/New Haven hospital for 3 days of testing. Within that time, they were required to stay on the unit, within a similar controlled environment to that of the cocaine participants.

During the study, all participants were exposed to three imagery conditions (stress, drug/alcohol cue, and neutral/ relaxing). The conditions were presented across three separate consecutive testing days with only one stimulus presentation per day. Condition order was assigned randomly and counterbalanced. In the case of the cocaine patients, imagery script development and laboratory sessions were conducted approximately 14 days after admission to the in-patient unit to allow for normalization of neurobiological changes associated with acute cocaine abstinence and adaptation to decreased access to nicotine smoking. All imagery scripts for the socially drinking controls were prepared before their admission to the GCRC. Research staff conducting the experiments were blind to imagery condition and the content of the scripts assigned to each laboratory session. Subjects also remained blind until imagery presentation.

\section{Imagery Script Development and Training}

Briefly, the stress imagery script was based on each individual subjects' description of a recent personal stressful event that was experienced as most stressful. Most 
stressful was determined by having each subject rate their perceived stress on a 10 -point Likert scale where $1=$ not at all stressful and $10=$ the most stress they felt recently in their life. Only situations rated as 8 or above on the 10-point scale were accepted as appropriate for script development. These ratings ensured individual calibration of level of perceived stress across subjects and groups. Also, no trauma-related stressful situations were allowed. Examples of stress scripts included breaking up or arguing with significant others and unemployment-related stress such as being fired or laid off from work. The drug/alcohol cue scripts were developed by having subjects identify a recent personal situation that included drug-related stimuli and resulted in subsequent cocaine use, in the case of the cocaine patients, and alcohol use in the case of the socially drinking controls. This might include either being at a bar or watching others smoke crack and drink alcohol. A neutral script was developed from the subjects' description of a personal non-drug-related neutral-relaxing situation. Examples of neutral relaxing situations include a summer beach scene or a relaxing fall afternoon reading. All scripts were then recorded onto an audiotape to be played in the laboratory sessions.

On a day before the laboratory sessions, subjects were brought into the testing room to acclimatize them to specific aspects of the study procedures including the subjective rating forms and training in relaxation and imagery procedures, as described previously in Sinha et al (2003).

\section{Laboratory Sessions}

On each testing day, subjects abstained from breakfast and were brought into the testing room at 0745 . All subjects were allowed an initial smoke break at 0730 to address nicotine craving. A blood pressure cuff was placed on the subject's preferred arm to monitor blood pressure (SBP and DBP) and a pulse sensor was placed on the subject's forefinger to obtain a measure of heart rate. Self-reports of craving and anxiety were completed after set-up. This was followed by a 1-h adaptation period during which the subjects were instructed to practice relaxation. At 0900, subjects were provided with headphones and were given the following instructions for the imagery procedure: 'Close your eyes and imagine the situation being described, 'as if it were happening right now. Let your body and mind get completely involved in the situation, doing what you would do in the real situation'. The length of each script was approximately $5 \mathrm{~min}$. Heart rate and blood pressure were continuously monitored during the imagery period.

Craving, emotion ratings, heart rate, and blood pressure were measured straight after set-up (0 time point), at two baseline time points (one $45 \mathrm{~min}$ after set-up ( + 45 time point) and one $65 \mathrm{~min}$ after set-up (+65 time point)), immediately following imagery ( +77 time point), and periodically every $15 \mathrm{~min}$ after the imagery period $(+90,+105,+120$, and +135 time points $)$. If participants' anxiety remained above baseline levels at the final time point, they were taken through another series of relaxation procedures until their ratings returned to baseline levels. After the final assessments, the blood pressure cuff and pulse sensor were removed and breakfast was served.

\section{Laboratory Assessments}

Subjective measures: drug/alcohol craving. Craving was measured by desire for each group's preferred drug. Cocaine patients rated desire for cocaine while social drinkers rated craving for alcohol. In addition, alcohol craving ratings were also obtained in the cocaine patients as they also consumed significantly greater amounts of alcohol in the 30 days before in-patient treatment compared with the socially drinking controls (Table 1). The desire to use cocaine was assessed using a 10-point visual analog scale (VAS) in which $0=$ not at all and $10=$ more than ever. Participants were requested to rate the intensity of desire for using cocaine at that moment. Alcohol craving: The desire to use alcohol was also assessed using a similar VAS anchored as above. Anxiety: Participants were requested to rate how tense, anxious, and/or jittery they felt at that moment using a VAS, again anchored as above.

Subjective measures: Differential Emotion Scale (DES). The DES (Izard, 1972) is a common measure of emotional experience and has been validated across a wide range of populations (Boyle, 1984; Fuenzalida et al, 1981). The scale has also been used to assess emotions in several drugabusing populations (Sinha et al, 1999, 2000, 2003; Hyman et al, 2007a; Fox et al, 2007). The scale comprises 30 emotional words (or items) and participants were required to rate on a 5-point scale the extent to which each word described the way he/she felt at the current time. The scale was then collapsed into the following subscales: anger, fear, sadness, joy, and neutral-relaxed feelings.

Physiological measures. A Critikon Dinamap 120 Patient Monitor was used to assess blood pressure. A pulse sensor was attached to the subject's finger and connected to the Dinamap Monitor to provide a continuous measure of pulse.

\section{Statistical Analysis}

Linear mixed effect (LME) models (Laird and Ware, 1982) were implemented to analyze the data using SAS software (version 9.1; SAS Institute, Cary, NC, USA). We first examined group and gender differences at baseline for each measure. The within-subjects factors of condition-3 (stress, drug cue, and neutral) and the between-subjects factors of drug group-2 (cocaine patients and social drinkers) and gender-2 (males and females) were the fixed effects. Subjects represented the random effects factor. To assess response to stress, drug/alcohol cues, and neutral cues, the within-subjects factors of condition-3 (stress, drug cue, and neutral) and time point-5 or -4 (varying levels) and the between-subjects factors of drug group-2 (cocaine patients and social drinkers) and gender-2 (males and females) were the fixed effects. Subjects again represented the random effect. In the case of baseline differences for any particular measure, the baseline response $(+65)$ was included as a random effect (covariate) in the LME model.

A series of Spearman's rho correlations were also performed to assess the associations between drug/alcohol craving and all subjective and physiological measures. All measures were averaged across time points for each condition. Wilcoxon rank sum tests and $\chi^{2}$ analyses were 
Table I Showing Demographics and Drug Use Variables for Cocaine Abusing and Socially Drinking Control Males and Females

\begin{tabular}{|c|c|c|c|c|c|}
\hline Subject variable & $\begin{array}{l}\text { Cocaine females } \\
\qquad(n=17)\end{array}$ & $\begin{array}{l}\text { Cocaine males } \\
\qquad(n=23)\end{array}$ & $\begin{array}{l}\text { Healthy females } \\
\qquad(n=19)\end{array}$ & $\begin{array}{l}\text { Healthy males } \\
\quad(n=2 I)\end{array}$ & $\begin{array}{c}p \text {-values for } \\
\chi^{2}\end{array}$ \\
\hline Race & & & & & $<0.2$ \\
\hline African American & 6 & 12 & 8 & 2 & \\
\hline Hispanic & । & 2 & 2 & 5 & \\
\hline Years of education & $13.53(1.33)$ & $13.78(2.11)$ & |3.89(3.21) & $14.29(1.55)$ & $<0.4$ \\
\hline Cocaine use (no. of days used in past 30 ) & I $7.35(10.05)$ & I6.96 (8.35) & $0(0)$ & $0(0)$ & $<0.0001$ \\
\hline Alcohol use (no. of days used in past 30 ) & $7.7 \mid(8.45)$ & I $1.00(10.55)$ & $2.16(2.7 I)$ & $7.24(8.00)$ & $<0.02$ \\
\hline Years of cocaine use & $9.12(6.43)$ & $8.56(7.00)$ & 1.00 & $1.00(1.41)$ & $<0.1$ \\
\hline Years of alcohol use & $9.71(8.26)$ & $13.29(10.19)$ & $5.59(6.00)$ & $11.42(10.44)$ & $<0.07$ \\
\hline
\end{tabular}

used to assess both demographic differences and drug use differences between the groups and across gender.

\section{RESULTS}

\section{Participants}

All groups were statistically matched for age, race, and years of education. All cocaine patients and social drinking males consumed significantly more alcohol compared to social drinking females during the 30 days before study participation. Lifetime prevalence of DSM-IV mood disorders and PTSD were also significantly higher in the cocaine patients compared with the social drinkers. Demographics and drug use data are shown in Table 1.

\section{Baseline Findings}

Cocaine patients reported significantly higher basal levels of drug/alcohol craving $(1,76=5.5 ; p=0.02)$, anger $(1,76=5.5 ; p=0.02)$, anxiety $(1,76=13.0 ; p=0.0006)$, and sadness $(1,76=5.0 ; p<0.03)$ compared with social drinkers. Significantly higher baseline sadness $(1,76=4.2$; $p=0.04)$, and $\operatorname{SBP}(1,76=6.0 ; p<0.02)$ was also observed in males compared to females.

\section{Response Findings}

$\alpha$-Values for all significant two- and three-way simple effect contrasts are presented in the text and figures. In the case of significant baseline differences, baseline values for these specific measures were included as covariates.

\section{Effects of Condition}

Significant effects of condition were seen for drug/alcohol craving $(2,152=89.3 ; p<0.0001)$, alcohol craving $(2,152=$ $32.0 ; p<0.0001)$, anxiety $(2,151=137.5 ; p<0.0001)$, anger $(2,152=161.6 ; p<0.0001)$, fear $(2,152=48.4 ; p<0.0001)$, sadness $(2,152=172.4 ; p<0.0001)$, joy $(2,152=109.8$; $p<0.0001)$, relaxed state $(2,152=126.8 ; p<0.0001)$, heart rate $(2,152=5.3 ; p=0.006), \operatorname{SBP}(2,151=11.6 ; p<0.0001)$, and DBP $(2,152=4.8 ; p<0.01)$. Participants reported significantly greater drug/alcohol craving, alcohol craving, anxiety, anger, and sadness in the stress and drug cue compared with the neutral condition (see Figures 1 and 2). Significantly greater fear was reported in the stress compared to the neutral condition only $(p<0.0001)$. All participants also reported significantly greater drug/alcohol craving in the drug cue compared with the stress condition $(p=0.01)$ and significantly greater anxiety, anger, fear, and sadness in the stress compared with the cue condition $(p<0.0001$, in all cases; see Figures 1 and 2$)$. In relation to positive emotions, participants reported significantly greater joy and relaxed state in the neutral compared with both the stress and drug cue conditions and significantly greater joy and relaxed state in the drug cue compared with the stress condition ( $p<0.0001$, in all cases).

Participants demonstrated significantly higher heart rate, SBP, and DBP in the stress compared with the neutral condition (heart rate, $p=0.004$; SBP, $p=0.001$; DBP, $p=0.003)$. They also demonstrated significantly higher heart rate, SBP, and DBP in the cue compared with the neutral condition (heart rate, $p=0.008$; SBP, $p<0.006$; DBP, $p<0.03$; see Figure $1 c$ and $d)$.

\section{Main Effects (Drug Group)}

Main effects of group for drug/alcohol craving $(1,76=31.4$; $p<0.0001)$, alcohol craving $(1,76=7.6 ; p=0.007)$, anxiety $(1,76=17.6 ; p<0.0001)$, anger $(1,76=13.4 ; p=0.0005)$, fear $(1,76=5.9 ; p<0.02)$ and sadness $(1,76=13.1$; $p=0.0005)$ indicated that cocaine patients reported significantly higher scores for each of these measures compared with social drinkers (see Figures 1 and 2).

\section{Main Effects (Gender)}

A main effect of gender for heart rate $(1,76=3.9 ; p=0.05)$ indicated that females demonstrated significantly higher 


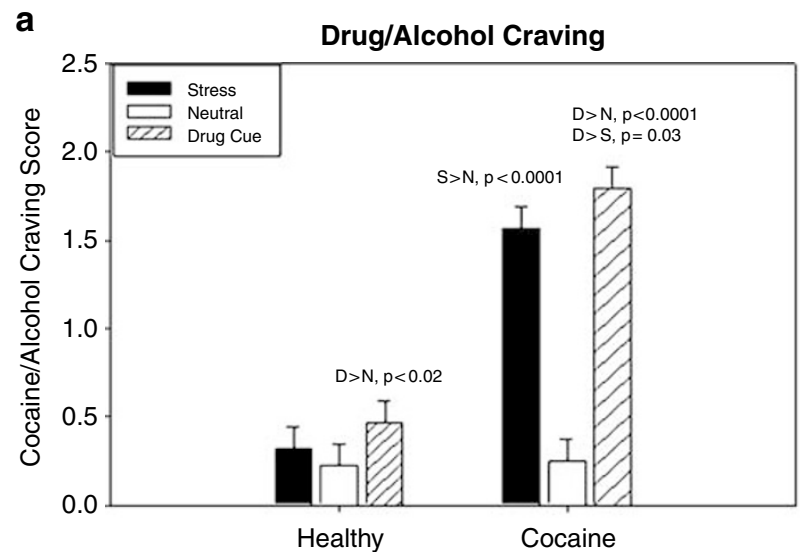

b
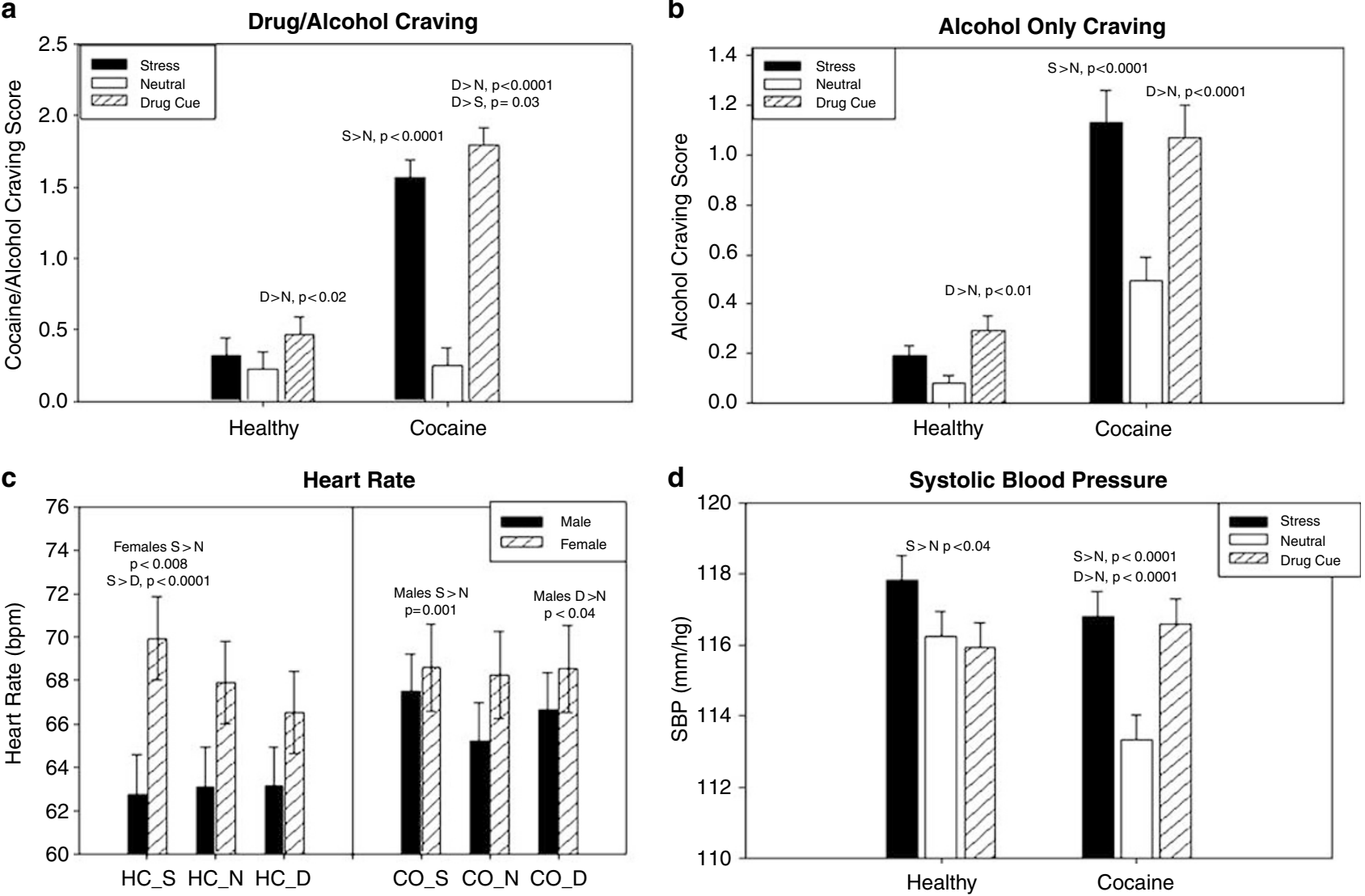

HC Females $>$ HC Males in Stress $(p<0.007)$

Figure I Group and gender differences in craving and cardiovascular response. (a) Drug/alcohol craving. All means are adjusted for baseline differences and data are collapsed across all time points. $\mathrm{Coc}>\mathrm{HC}$ in stress, $p<0.000 \mathrm{I}$; Coc $>\mathrm{HC}$ in drug cue, $p<0.000 \mathrm{I}$. (b) Alcohol craving. All means are adjusted for baseline differences and data are collapsed across all time points. Coc $>\mathrm{HC}$ in stress, $p=0.005$; Coc $>\mathrm{HC}$ in drug cue, $p<0.004$. (c) Heart rate. All means are adjusted for baseline differences and data are collapsed across all time points. Cocaine males $>$ healthy males in stress, $p<0.06$; control females $>$ control males in stress, $p<0.007$. In cocaine males: $S>N, p=0.00$ I; $D>N, p<0.04$. In healthy females: $S>N, p<0.008 ; S>D, p<0.000$ I. (d) Systolic blood pressure. All means are adjusted for baseline differences and data are collapsed across all time points.

pulse rate compared with males across all three imagery conditions (see Figure 1c).

\section{Interaction Effects (Drug Group $\times$ Condition)}

Significant interactions were observed for drug/alcohol craving $(2, \quad 152=53.4 ; p<0.0001)$, alcohol craving $(2$, $152=12.6 ; p<0.0001)$, anxiety $(2,151=7.0 ; p<0.0001)$, anger $\quad(2, \quad 152=18.2 ; p<0.0001)$, fear $(2,152=6.6$; $p<0.002)$, sadness $(2,152=15.9 ; p<0.0001)$, joy $(2$, $152=5.7 ; p=0.004)$, and SBP $(2,152=5.9 ; p<0.004)$. With regard to craving, anxiety, and DES rating scales, cocaine patients demonstrated significantly greater responses to the stress and the drug/alcohol cue imagery conditions compared with social drinkers. In the cocaine group, significantly greater anxiety, anger, fear, and sadness were seen in response to the cue compared with the neural imagery condition $(p<0.0001$, in all cases; see Figure 2$)$. Importantly, these enhanced negative emotional responses to drug and alcohol cues were not observed in the social drinkers. Similarly, cocaine patients also reported significantly increased drug/alcohol craving and alcohol craving in response to the stress compared with the neutral imagery condition $(p<0.0001$, in both cases). Again, this signifi- cantly increased craving response to stress was not observed in the social drinkers. Both groups reported increased drug/ alcohol craving and increased alcohol craving in the cue compared with the neutral imagery condition (see Figure 1a and $\mathrm{b}$ ), although cocaine patients reported significantly higher cue-induced drug/alcohol craving $(p<0.0001)$ and alcohol craving $(p<0.004)$ compared with controls. In relation to positive affect, cocaine patients reported a significantly greater decrease in joy in the cue compared with the neutral condition $(p<0.0001)$. In controls, joyrelated differences between these two conditions were not as strong $(p<0.05)$.

Cocaine patients also demonstrated significantly enhanced SBP following both the stress and cue conditions compared with the neutral imagery condition $(p<0.0001$, in both cases). In the social drinkers, only a stress-related increase in SBP was observed $(p<0.04$; see Figure 1d).

\section{Interaction Effects (Gender $\times$ Condition)}

Significant interactions were observed for anxiety (2, $152=15.3 ; p<0.0001)$, anger $(2,152=4.1 ; p<0.02)$, fear $(2,152=5.4 ; p=0.005)$, sadness $(2,152=8.0 ; p=0.0005)$, 
a Anxiety Scale

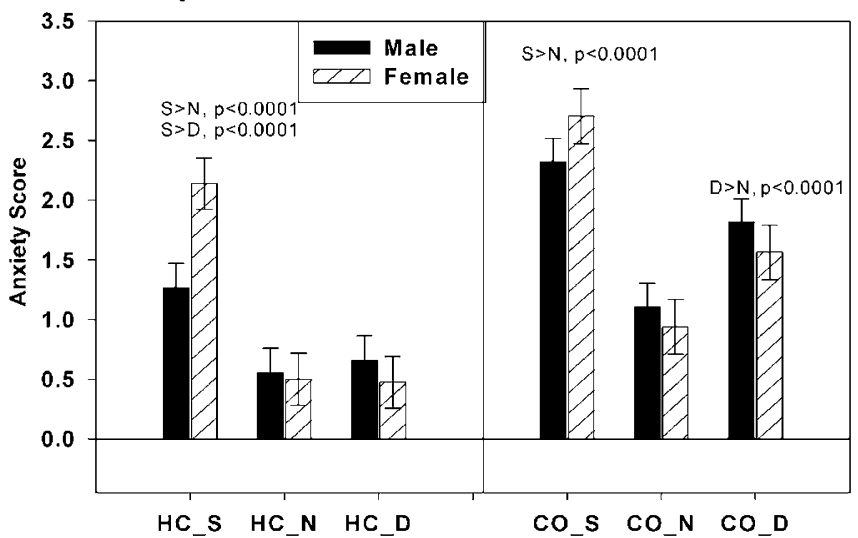

c

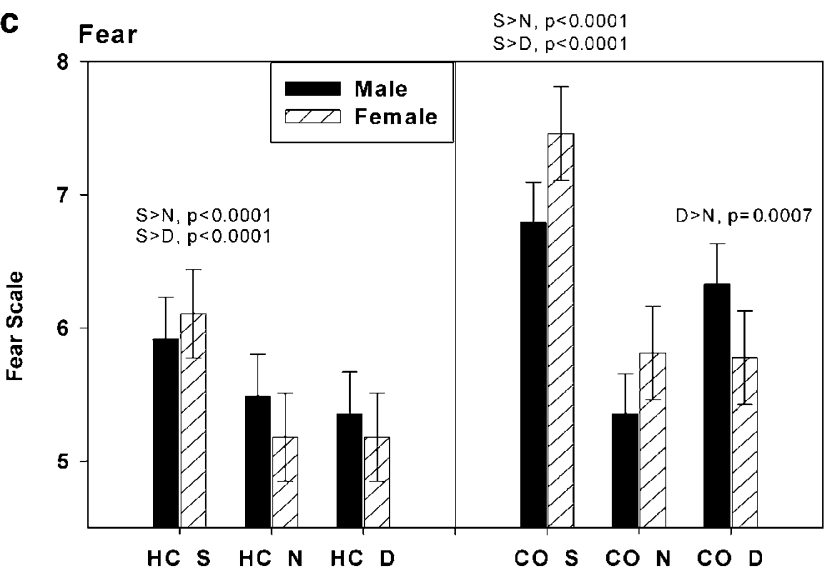

b Anger
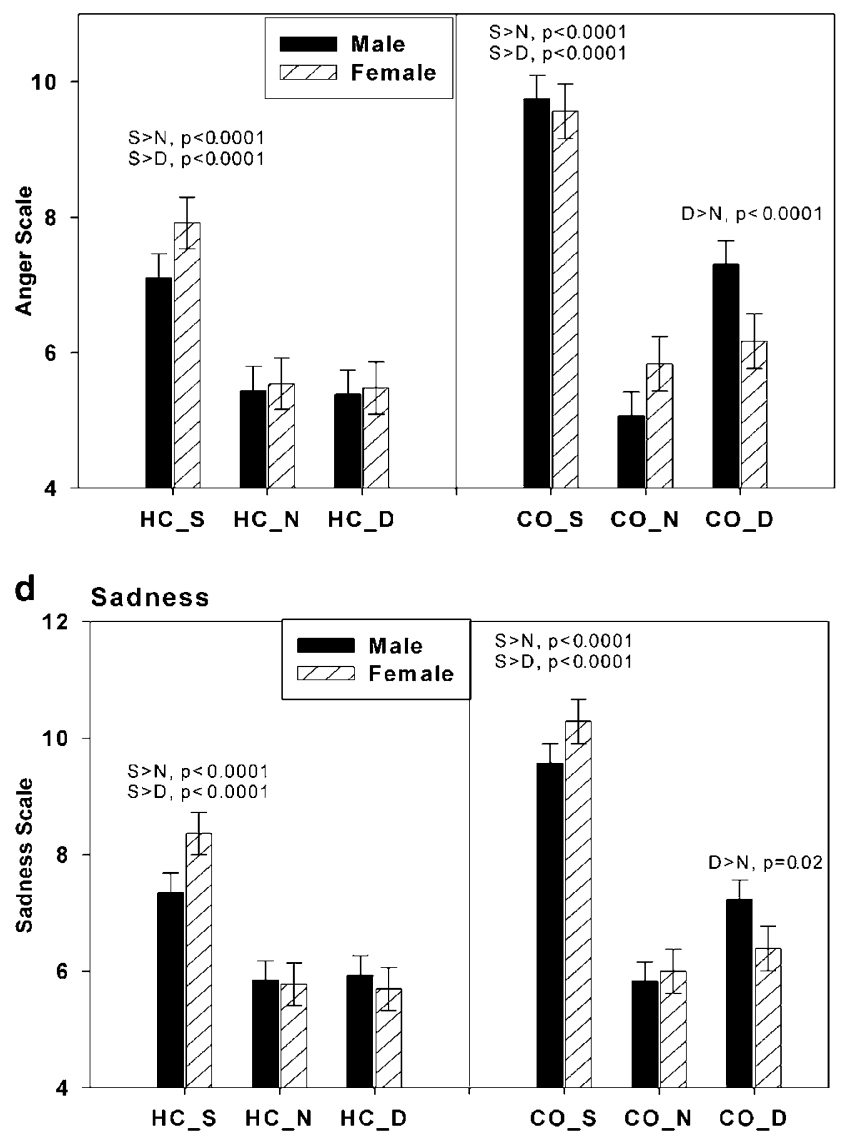

Figure 2 Group and gender differences in anxiety and negative emotion. (a) Anxiety scale. All means are adjusted for baseline differences and data are collapsed across all time points. Coc $>\mathrm{HC}$ in stress, $p<0.000 \mathrm{I}$; Coc $>\mathrm{HC}$ in drug cue, $p<0.000 \mathrm{I}$. In males: $\mathrm{S}>\mathrm{N}, p<0.000 \mathrm{I} ; \mathrm{S}>\mathrm{D}, p=0.00 \mathrm{I}$; $\mathrm{D}>\mathrm{N}, p<0.000 \mathrm{I}$. In females: $\mathrm{S}>\mathrm{N}, p<0.000 \mathrm{I}$; $\mathrm{S}>\mathrm{D}, p=0.000 \mathrm{I}$; $\mathrm{D}>\mathrm{N}, p<0.000 \mathrm{I}$. (b) Anger. All means are adjusted for baseline differences and data are collapsed across all time points. Coc $>\mathrm{HC}$ in stress, $p<0.000 \mathrm{l}$; Coc $>\mathrm{HC}$ in drug cue, $p=0.0006$. In males: $\mathrm{S}>\mathrm{N}, p<0.000 \mathrm{I}$; $\mathrm{S}>\mathrm{D}$, $p<0.000$ I; $D>N, p<0.000$ I. In females: $S>N, p<0.000$ I; $S>D, p<0.000$ I. (c) Fear. All means are adjusted for baseline differences and data are collapsed across all time points. Coc $>\mathrm{HC}$ in stress, $p=0.0008$; Coc $>\mathrm{HC}$ in drug cue, $p<0.02$. In males: $\mathrm{S}>\mathrm{N}, p<0.000 \mathrm{I} ; \mathrm{S}>\mathrm{D}, p<0.04$; $\mathrm{D}>\mathrm{N}$, $p<0.000$ I. In females: $S>N, p<0.000$ I; $S>$ D, $p<0.000$ I. (d) Sadness. All means are adjusted for baseline differences and data are collapsed across all time points. Coc $>\mathrm{HC}$ in stress, $p<0.000 \mathrm{I}$; Coc $>\mathrm{HC}$ in drug cue, $p<0.006$. In males: $\mathrm{S}>\mathrm{N}, p<0.000 \mathrm{I} ; \mathrm{S}>\mathrm{D}, p<0.000 \mathrm{I} ; \mathrm{D}>\mathrm{N}, p<0.002$. In females: $S>N, p<0.0001 ; S>D, p=0.0001$.

and relaxed state $(2,152=4.8 ; p<0.01)$. The interaction for DBP approached statistical significance $(2,152=3.0$; $p<0.06)$. Females reported significantly greater anxiety $(p<0.004)$, and sadness $(p<0.02)$ compared with males in the stress imagery condition. However, only males demonstrated an enhanced negative emotional response to cue and reported significantly greater anger $(p<0.0001)$, fear $(p<0.0001)$ and sadness $(p<0.002)$ in the cue compared with the neutral condition. Both males and females reported significantly higher anxiety, anger, fear, and sadness in the stress compared with the neutral and cue conditions $(p<0.0001$, in all cases; see Figure 2$)$. In relation to positive affect, males reported a significantly greater decrease in relaxed state in the cue compared with the neutral condition $(p<0.0001)$. In females, differences in relaxed state between these two conditions were less robust $(p<0.01)$.

Males also demonstrated higher DBP in the drug cue condition compared with the neutral condition $(p=0.03)$. This condition difference was not observed in the females. Extended analyses also showed that this effect was driven by cocaine-abusing males who demonstrated significantly increased DBP compared with cocaine-abusing females in the drug cue condition $(p<0.04)$. This effect of gender on cue-related DBP response was not observed in the social drinkers.

\section{Interaction Effects (Drug Group $\times$ Gender $\times$ Condition)}

Significant interactions were observed for anger (2, $152=4.0 ; p=0.02)$, fear $(2,152=3.0 ; p=0.05)$, and heart rate $(2,152=6.0 ; p=0.003)$. Cocaine-abusing males demonstrated an increased anger and fear response following both the stress (anger, $p<0.0001$; fear, $p<0.05$ ), and cue (anger, $p=0.0002$; fear, $p<0.03$ ) imagery conditions compared with the control males. However, cocaineabusing females showed an enhanced anger and fear response compared with control females (anger, $p=0.003$; fear, $p<0.006)$ only in the stress condition and not the cue condition. Furthermore, cocaine-abusing males reported significantly greater cue-induced anger compared with 
cocaine-abusing females $(p=0.03)$, with no differences observed between gender in the controls. Only the cocaine-abusing males demonstrated a significantly increased anger and fear response to the cue imagery compared to the neutral imagery condition (anger, $p<0.0001$; fear, $p<0.0001$; see Figure $2 \mathrm{~b}$ and $c$ ).

Cocaine-abusing males also demonstrated a significantly greater heart rate response to both stress $(p=0.001)$ and cue $(p<0.04)$ compared with the neutral imagery condition. This was not observed in the control males or in the cocaineabusing females. Female controls demonstrated only a stressrelated increase in heart rate compared with both the neutral $(p<0.008)$ and drug cue $(p<0.0001)$ imagery conditions, and these increases were significantly greater than the stressrelated HR response in the male controls $(p<0.007)$. This stress-related gender difference was not seen in cocaine patients. However, cocaine-abusing males showed an almost significant increase in stress-related heart rate response compared with control males $(p<0.06$; see Figure $1 c)$.

\section{Interaction Effects (Drug Group $\times$ Condition $\times$ Time Point)}

Significant interactions were observed for drug/alcohol craving $(10,754=3.9 ; p<0.0001)$, anxiety $(10,748=3.9$; $p<0.0001)$, anger $(8450=2.6 ; p<0.02)$, fear $(8602=2.1$; $p=0.03)$, and relaxed state $(8602=2.7 ; p<0.007)$, indicating that cocaine patients took significantly longer than controls to recover following exposure to stress- and cuerelated imagery. Cocaine patients reported significantly increased drug/alcohol craving at the imagery, initial, second, third, and fourth recovery time points compared with controls in both the stress and drug cue imagery conditions. These time point differences between groups were not observed in the neutral imagery condition. Cocaine patients also reported significantly increased anxiety at the imagery, initial, and second recovery time points compared with controls in the stress imagery condition. These time point differences between the groups were not observed in either the drug cue or the neutral imagery conditions. Cocaine patients also reported significantly higher anger and fear at the imagery, initial, and second recovery time points compared with controls in both the stress and cue conditions. Again, these time point differences between groups were not observed in the neutral condition. In relation to positive emotion, controls reported significantly greater relaxed state compared with the cocaine patients in the cue condition at the imagery time point $(p<0.003)$.

\section{Correlational Analyses}

In cocaine patients, moderate correlations were obtained between stress-induced craving and anxiety $(r=0.55$; $p=0.0002)$, anger $(r=0.47 ; p=0.002)$, fear $(r=0.33$, $p<0.04)$, and sadness $(r=0.35, p=0.02)$ as well as cueinduced craving and anxiety $(r=0.67 ; p<0.0001)$, anger $(r=0.43 ; p=0.006)$, fear $(r=0.54, p=0.0003)$, and sadness $(r=0.47, p=0.002)$.

In social drinkers, a significant correlation was observed between stress-induced craving and anger only $(r=0.32$; $p=0.04$ ) and cue-induced craving and anxiety only
( $r=0.51 ; p=0.0008)$. No significant correlations were observed for either groups between craving and physiological measures for the stress or the drug cue condition.

\section{Post hoc Secondary Analyses Accounting for Childhood Trauma, Psychiatric Co-morbidity, and Smoking History}

Trauma- and anxiety-related disorders have often been associated with hyper-responsivity to trauma-related and non-trauma-related startle stimuli (Pitman et al, 1987; Casada et al, 1998). Although trauma-related cues were specifically not used in the scripts, cocaine patients reported a higher lifetime prevalence of major depression and PTSD (see Table 1) and are known to have greater childhood trauma history (Hyman et al, 2007b). We therefore reanalyzed the above findings excluding individuals with lifetime depression (cocaine, $n=14$ ) and those with lifetime PTSD (cocaine, $n=13$; controls, $n=3$ ). To ensure that childhood trauma was also not impacting on stress- and cue-related responsivity, a correlational analysis was performed in the cocaine sample $(n=40)$ assessing the association between trauma (Childhood Trauma Questionnaire (CTQ); Bernstein and Fink, 1998) and laboratory response measures.

Although attempts were made in the current study to avoid nicotine withdrawal by providing participants with four regular smoke breaks per day and allowing them to smoke before the laboratory study, it was still important to ensure that nicotine craving was not having an impact on stress- or cue-related response. Non-parametric analysis was therefore used to assess any potential variation in laboratory response measures (averaged across time points) between cocaine-dependent smokers $(n=33)$ and nonsmokers $(n=6)$.

In the cocaine patients, no significant associations were seen between the CTQ and stress- or cue-induced craving, anxiety, heart rate, SBP, or DBP either at peak or averaged across time points. Results also remained similar once participants with lifetime depression and PTSD had been removed from the LME analyses. Findings still indicated that cocaine patients reported significantly increased craving and negative emotions in the stress and cue conditions compared with social drinkers. Cocaine-dependent non-smokers reported significantly increased drug and alcohol craving scores compared with cocaine-dependent smokers $(p=0.0004)$.

\section{DISCUSSION}

Findings from the current study are the first to confirm the model proposed in previous human research (Sinha et al, 2001, 2003; Fox et al, 2005) that there is a hyper-responsivity to stress and drug cue exposure that ensues during cocaine abstinence, and it is marked by enhanced drug craving and emotional and physical sensitivity to stress and drug cues. Both stress- and cueinduced cocaine craving was significantly associated with increased anxiety, anger, fear, and sadness in the cocaine patients. Notably, this was less apparent in the controls. Moreover, although social drinkers showed an appropriate 
stress-related increase in all negative, no significant increase in craving accompanied this response. Furthermore, these effects were more persistent, in that following exposure to both the stress and cue imagery, it took the cocaine patients significantly longer for the cocaine craving and negative emotion scores to recover and return to baseline compared to social drinkers. This hyper-responsiveness to stress and cue exposure in cocaine patients was not correlated with childhood trauma scores and remained significant even when patients with a lifetime prevalence of major depression and PTSD were excluded. Identifying the emotional components of the stress- and cue-related craving state is important as stress- and cue-induced craving and emotional response has been previously associated with a higher frequency of cocaine use (Fox et al, 2005) and with cocaine relapse outcomes (Sinha et al, 2006).

Findings also showed that the cocaine patients demonstrated a very different emotional response to drug and alcohol cues by reporting significantly higher craving, anxiety, anger, fear, and sadness ratings compared with social drinkers. In cocaine patients, cue-induced cocaine craving was significantly correlated with anxiety, anger, fear, and sadness. Although the social drinkers reported significant alcohol craving in the cue condition, they demonstrated no concomitant increases in anxiety, anger, fear, or sadness, only increases in a positive relaxed state. It is also interesting to note that alcohol cue-induced craving was only significantly associated with increases in anxiety rather than negative emotions, in social drinkers. This combination of both jitteriness and relaxed mood possibly representative of a hedonic arousal state following cue exposure, is consistent with previous research indicating that stress markers may mediate the rewarding properties of drugs (Oswald et al, 2005; Mendelson et al, 2005). Importantly, however, the emotional response to alcohol cues in the social drinkers was markedly different from the range of negative emotions reported following cue exposure in the cocaine participants.

In line with these group differences in cue-induced emotional response, cocaine patients also demonstrated significant cue-related increases in SBP and heart rate that was not observed in social drinkers. Stress and cue-related increases in heart rate and blood pressure have been observed alongside increases in cocaine craving within recently abstinent cocaine abusers in several studies (Back et al, 2005; Sinha et al, 2001, 2003; Fox et al, 2005, 2006). $\beta$-Adrenergic blockade by drugs such as propranolol have also been shown to reduce the symptoms of autonomic arousal associated with early cocaine abstinence and improve treatment outcome (Kampman et al, 2001b, 2006). It may therefore be the case that increased heart rate and blood pressure represent a non-specific physiological marker of stress- and cue-induced craving state that may be elevated during cocaine abstinence. Although no association was seen between these physiological measures and subjective craving-related states, the disparity between subjective and physiological data is not uncommon and has often been discussed in stress studies (Cohen et al, 1995; Back et al, 2005). We have previously suggested that stress- and cuerelated subjective and physiological responses represent semiindependent systems impacting different aspects of adaptation to stressful situations (see Sinha et al, 2003 for review).
The qualitatively different emotional and physiological response to stress- and cue-related craving seen between the cocaine patients and social drinkers provides support for the notion of a potential shift in hedonic set-point (Ahmed and Koob, 2005). The findings suggest an increase in the salience of distress-related signaling possibly due to a potential underactivation of reward circuits and overactivation of stress systems during early abstinence after chronic cocaine abuse (Koob and Le Moal, 1997, 2001). Neuroadaptions associated with the stress and reward pathways could explain this increased craving-related arousal state. For example, research has reported significantly decreased levels of extracellular serotonin and dopamine in the nucleus accubens (NAC) following continuous cocaine intake (Parsons et al, 1996; Weiss et al, 1992; Mateo et al, 2005) and increased levels of extracellular CRF following abstinence (Richter et al, 1995; Sarnyai, 1998). Human studies have also shown decreased D2 receptors following cocaine withdrawal and up to 3 months of cocaine abstinence (Volkow et al, 1993, 1996).

A significant effect of gender on stress- and cue-related physiological and emotional response was also observed. Socially drinking females demonstrated significantly increased heart rate following exposure to the stress imagery condition compared with socially drinking males. Notably, this gender difference was not observed in the cocaine abusers, possibly owing to the fact that the cocaine-abusing males demonstrated an increased response to stress similar to the increased response levels observed in the cocaineabusing females. Furthermore, gender-related dissociations in stress and drug cue exposure with regard to subjective emotional response were also observed. All females reported significantly higher stress-induced anxiety and sadness compared with males, whereas cocaine-abusing males appeared to be more emotionally and physiologically reactive in the drug cue condition compared with cocaineabusing females. Males reported significantly higher anxiety, anger, fear, and sadness following the drug cue condition compared with the neutral imagery condition, as well as significantly decreased relaxed state in the cue compared with the neutral condition.

Higher stress-induced anxiety and sadness in all females are consistent with previous work suggesting greater susceptibility to sadness and anxiety in women (Brody, 1999; Eisenberg et al, 1991) and greater anxiety in cocainedependent females (Back et al, 2005). Our findings that men were more reactive to the cue condition than females are consistent with some previous research. Sterling et al (2004) showed that males were significantly more physiologically reactive to cocaine-related cues and triggers than females, while other studies have shown no gender variation (Robbins et al, 1999; Avants et al, 1995). In this study, increased cue reactivity in males (and more specifically cocaine-abusing males) was a robust finding and is supported by neuroimaging research where males showed greater cue-induced activation of paralimbic regions associated with automatic emotional processing (Li et al, 2005). As such, males may demonstrate greater emotional and physiological arousal than females to more conditioned appetitive stimuli such as drug cues.

Limitations of the current study should be acknowledged. While comparable individually calibrated stressors were 
used and cocaine patients showed greater stress responses, it is possible that drug and alcohol cue exposure scenarios are largely positive in nature in social drinkers, while such situations are more distressing for abstinent cocaine patients. Nonetheless, cocaine patients showed comparable levels of enhanced sensitivity in response to stress and drug cue exposure. It is also important to note that a higher number of cocaine patients had a smoking history compared to social drinkers. However, non-smoking cocaine patients had higher stress- and cue-induced drug craving levels compared to nonsmoking cocaine patients, suggesting that increased sensitivity to drug and alcohol craving in the current study was not a product of nicotine withdrawal.

In summary, findings from this study have shown that, compared with social drinkers, recently abstinent cocainedependent individuals demonstrated an enhanced sensitivity to negative emotions, physiological output, and cocaine craving following both stress and drug cue imagery exposure. Consistent with previous research showing that stress-induced craving and related arousal predicts relapse outcomes (Sinha et al, 2006), these data also underscore the need to address this enhanced sensitivity to stress- and cueinduced craving in pharmacological and behavioral treatment development for cocaine relapse prevention. Findings have also indicated that stress- and cue-induced craving may represent an emotionally and physiologically different state in males and females, highlighting the importance of developing more specialized gender-related treatment interventions for cocaine dependence.

\section{ACKNOWLEDGEMENTS}

This study was supported in part by Grants R01-DA11077 (Sinha), P50-DA16556 (Sinha), R0I-AA13892 (Sinha), K02-DA17232 (Sinha), and P50-DA16556 (Sinha) from the National Institutes of Health, Bethesda, MD, USA. We thank the staff at the Substance Abuse Treatment Unit, Clinical Neuroscience Research Unit, and the General Clinical Research Center at Yale University School of Medicine for their assistance in completing these studies.

\section{DISCLOSURE/CONFLICT OF INTEREST}

The authors declare that they have no competing financial interests.

\section{REFERENCES}

Adinoff B, Junghanns K, Kiefer F, Krishnan-Sarin S (2005). Suppression of the HPA axis stress-response: implications for relapse. Alcohol Clin Exp Res 30: 585.

Ahmed SH, Koob GF (2005). Transition to drug addiction: a negative reinforcement model based on an allostatic decrease in reward function. Psychopharmacology (Berl) 180: 473-490.

Avants SK, Margolin A, Kosten TR, Cooney NL (1995). Differences between responders and nonresponders to cocaine cues in the laboratory. Addict Behav 20: 215-224.

Back SE, Brady KT, Jackson JL, Salstrom S, Zinzow H (2005). Gender differences in stress reactivity among cocaine-dependent individuals. Psychopharmacology (Berl) 180: 169-176.
Bernstein DP, Fink L (1998). Childhood Trauma Questionnaire: A Retrospective Self-Report Manual. The Psychological Corporation: San Antonio, TX.

Beveridge TJ, Smith HR, Nader MA, Porrino LJ (2005). Effects of chronic cocaine self-administration on norepinephrine transporters in the nonhuman primate brain. Psychopharmacology (Berl) 180: 781-788 [E-pub 2005 Sep 14].

Boyle GJ (1984). Reliability and validity of Izard's differential emotion scale. Pers Individ Dif 5: 747-750.

Brady KT, Back SE, Waldrop AE, McRae AL, Anton RF, Upadhyaya $\mathrm{HP}$ et al (2006). Cold pressor task reactivity: predictors of alcohol use among alcohol-dependent individuals with and without comorbid posttraumatic stress disorder. Alcohol Clin Exp Res 30: 938-946.

Breese GR, Chu K, Dayas CV, Funk D, Knapp DJ, Koob GF et al (2005). Stress enhancement of craving during sobriety: a risk for relapse. Alcohol Clin Exp Res 29: 185-195.

Brody L (1999). Gender, Emotion, and the Family. Harvard University Press: Cambridge, MA.

Brunova J (2004). Endocrine diseases and diabetes. Cas Lek Cesk 143: $507-511$.

Callahan D, Cisin IH (1968). American drinking practices: summary of findings from a national probability sample. I. Extent of drinking by population subgroups. Q Journal Stud Alcohol 29: 130.

Casada JH, Amdur R, Larsen R, Liberzon I (1998). Psychophysiologic responsivity in posttraumatic stress disorder: generalized hyperresponsivity versus trauma specificity. Soc Biol Psyc 44: 1037-1044.

Cohen S, Kessler RC, Gordon LU (1995). Strategies for measuring stress in studies of psychiatric and physical disorders. In: Cohen S, Kessler RC, Gordon LU (eds). Measuring Stress: a Guide for Health and Social Scientists. Oxford University Press: New York. pp 3-26.

Cooney NL, Gillespie RA, Baker LH, Kaplan RF (1987). Cognitive changes after alcohol cue exposure. J Consult Clin Psychol 55: 150-155.

Eisenberg N, Fabes RA, Schaller M, Miller P, Carlo G, Poulin R et al (1991). Personality and socialization correlates of vicarious emotional responding. J Pers Soc Psychol 61: 459-470.

Erb S, Funk D, Le AD (2003). Prior, repeated exposure to cocaine potentiates locomotor responsivity to central injections of corticotropin-releasing factor (CRF) in rats. Psychopharmacology (Berl) 170: 383-389.

First MB, Spitzer RL, Gibbon M, Williams JBW (1997). Structured Clinical Interview for the DSM-IV Axis I Disorders-Patient Edition (SCID-I/P, Version 20, 4/97 Revision). New York State Psychiatric Institute: New York.

Fox HC, Berquist KL, Hong KI, Sinha R (2007). Stress-induced and alcohol cue-induced craving in recently abstinent alcohol dependent individuals. Alcoholism: Clin Exp Res 31: 395-403.

Fox HC, Garcia M, Kemp K, Milivojevic V, Kreek MJ, Sinha R (2006). Gender differences in cardiovascular and corticoadrenal response to stress and drug-cue in cocaine dependent individuals. Psychopharmacology 185: 348-357.

Fox HC, Talih M, Malison R, Anderson GM, Kreek MJ, Sinha R (2005). Frequency of recent cocaine and alcohol use affects drug craving and associated responses to stress and to drug-related cues. Psychoneuroendocrinology 30: 880-891.

Fuenzalida C, Emde RN, Pannabecker BJ, Stenberg C (1981). Validation of the differential emotion scale in 613 mothers. Motivation Emotion 5: 37-45.

Greeley JD, Swift W, Prescott J, Heather N (1993). Reactivity to alcohol-related cues in heavy and light drinkers. J Stud Alcohol 54: $359-368$.

Haile CN, GrandPre T, Kosten TA (2001). Chronic unpredictable stress, but not chronic predictable stress, enhances the sensitivity to the behavioral effects of cocaine in rats. Psychopharmacology (Berl) 154: 213-220. 
Hyman S, Fox HC, Hong K, Doebrick C, Sinha R (2007a). Stress and drug cue-induced craving in opioid dependent individuals in naltrexone treatment. Exp Clin Psychopharmacol 15: 134-143.

Hyman SM, Paliwal P, Sinha R (2007b). Childhood maltreatment, perceived stress, and stress-related coping in recently abstinent cocaine dependent adults. Psychol Addict Behav (in press).

Izard C (1972). Patterns of Emotions: A New Analysis of Anxiety and Depression. Academic Press: New York.

Kalivas PW, Stewart J (1991). Dopamine transmission in the initiation and expression of drug- and stress-induced sensitization of motor activity. Brain Res Brain Res Rev 16: 223-244.

Kampman KM, Alterman AI, Volpicelli JR, Maany I, Muller ES, Luce DD et al (2001a). Cocaine withdrawal symptoms and initial urine toxicology results predict treatment attrition in outpatient cocaine dependence treatment. Psychol Addict Behav 15: 52-59.

Kampman KM, Dackis C, Lynch KG, Pettinati H, Tirado C, Gariti $\mathrm{P}$ et al (2006). A double-blind, placebo-controlled trial of amantadine, propranolol, and their combination for the treatment of cocaine dependence in patients with severe cocaine withdrawal symptoms. Drug Alcohol Depend 85: 129-137.

Kampman KM, Volpicelli JR, McGinnis DE, Alterman AI, Weinrieb RM, D'Angelo L et al (1998). Reliability and validity of the Cocaine Selective Severity Assessment. Addict Behav 23: 449-461.

Kampman KM, Volpicelli JR, Mulvaney F, Alterman AI, Cornish J, Gariti P et al (2001b). Effectiveness of propranolol for cocaine dependence treatment may depend on cocaine withdrawal symptom severity. Drug Alcohol Depend 63: 69-78.

Kenny PJ, Chen SA, Kitamura O, Markou A, Koob GF (2006). Conditioned withdrawal drives heroin consumption and decreases reward sensitivity. J Neurosci 26: 5894-5900.

Kenny PJ, Markou A (2005). Conditioned nicotine withdrawal profoundly decreases the activity of brain reward systems. J Neurosci 25: 6208-6212.

Kilts CD, Gross RE, Ely TD, Drexler KP (2004). The neural correlates of cue-induced craving in cocaine-dependent women. Am J Psychiatry 161: 193-194.

Koob GF, Ahmed SH, Boutrel B, Chen SA, Kenny PJ, Markou A et al (2004). Neurobiological mechanisms in the transition from drug use to drug dependence. Neurosci Biobehav Rev 27: 739-749.

Koob GF, Le Moal M (1997). Drug abuse: hedonic homeostatic dysregulation. Science 278: 52-58.

Koob GF, Le Moal M (2001). Drug addiction, dysregulation of reward, and allostasis. Neuropsychopharmacology 24: 97-129.

Laird NM, Ware JH (1982). Random-effects models for longitudinal data. Biometrics 38: 963-974.

Lepsch LB, Gonzalo LA, Magro FJ, Delucia R, Scavone C, Planeta CS (2005). Exposure to chronic stress increases the locomotor response to cocaine and the basal levels of corticosterone in adolescent rats. Addict Biol 10: 251-256.

Li CS, Kemp K, Milivojevic V, Sinha R (2005). Neuroimaging study of sex differences in the neuropathology of cocaine abuse. Gend Med 2: 174-182.

Mateo Y, Lack CM, Morgan D, Roberts DC, Jones SR (2005). Reduced dopamine terminal function and insensitivity to cocaine following cocaine binge self-administration and deprivation. Neuropsychopharmacology 30: 1455-1463.

Mendelson JH, Sholar MB, Goletiani N, Siegel AJ, Mello NK (2005). Effects of low- and high-nicotine cigarette smoking on mood states and the HPA axis in men. Neuropsychopharmacology 30: 1751-1763.

Moore RJ, Vinsant SL, Nader MA, Porrino LJ, Friedman DP (1998). Effect of cocaine self-administration on dopamine D2 receptors in rhesus monkeys. Synapse 30: 88-96.
Nader MA, Daunais JB, Moore T, Nader SH, Moore RJ, Smith HR et al (2002). Effects of cocaine self-administration on striatal dopamine systems in rhesus monkeys: initial and chronic exposure. Neuropsychopharmacology 27: 35-46.

Oswald LM, Wong DF, McCaul M, Zhou Y, Kuwabara H, Choi L et al (2005). Relationships among ventral striatal dopamine release, cortisol secretion, and subjective responses to amphetamine. Neuropsychopharmacology 30: 821-832.

Pariante CM (2003). Depression, stress and the adrenal axis. J Neuroendocrinol 15: 811-812.

Parsons LH, Weiss F, Koob GF (1996). Serotonin1b receptor stimulation enhances dopamine-mediated reinforcement. Psychopharmacology (Berl) 128: 150-160.

Pitman RK, Orr SP, Forgue DF, de Jong JB, Claiborn JM (1987). Psychophysiologic assessment of posttraumatic stress disorder imagery in Vietnam combat veterans. Arch Gen Psychiatry 44: 970-975.

Richter RM, Pich EM, Koob GF, Weiss F (1995). Sensitization of cocaine-stimulated increase in extracellular levels of corticotropin-releasing factor from the rat amygdala after repeated administration as determined by intracranial microdialysis. Neurosci Lett 187: 169-172.

Robbins SJ, Ehrman RN, Childress AR, O'Brien CP (1999). Comparing levels of cocaine cue reactivity in male and female outpatients. Drug Alcohol Depend 53: 223-230.

Sarnyai Z (1998). Neurobiology of stress and cocaine addiction. Studies on corticotropin-releasing factor in rats, monkeys, and humans. Ann NY Acad Sci 851: 371-387.

Schulteis G, Markou A, Cole M, Koob GF (1995). Decreased brain reward produced by ethanol withdrawal. Proc Natl Acad Sci USA 92: $5880-5884$.

Sinha R (2001). How does stress increase risk of drug abuse and relapse? Psychopharmacology (Berl) 158: 343-359.

Sinha R, Catapano D, O’Malley S (1999). Stress-induced craving and stress response in cocaine dependent individuals. Psychopharmacology (Berl) 142: 343-351.

Sinha R, Fuse T, Aubin LR, O'Malley SS (2000). Psychological stress, drug-related cues and cocaine craving. Psychopharmacology (Berl) 152: 140-148.

Sinha R, Garcia M, Paliwal P, Kreek MJ, Rounsaville BJ (2006). Stress-induced cocaine craving and hypothalamic-pituitaryadrenal responses are predictive of cocaine relapse outcomes. Arch Gen Psychiatry 63: 324-331.

Sinha R, Talih M, Malison R, Cooney N, Anderson GM, Kreek MJ (2003). Hypothalamic-pituitary-adrenal axis and sympatho-adreno-medullary responses during stress-induced and drug cue-induced cocaine craving states. Psychopharmacology (Berl) 170: 62-72.

Sterling RC, Dean J, Weinstein SP, Murphy J, Gottheil E (2004). Gender differences in cue exposure reactivity and 9-month outcome. J Subst Abuse Treat 27: 39-44.

Volkow ND, Fowler JS, Wang GJ, Hitzemann R, Logan J, Schlyer DJ et al (1993). Decreased dopamine D2 receptor availability is associated with reduced frontal metabolism in cocaine abusers. Synapse 14: 169-177.

Volkow ND, Wang GJ, Fowler JS, Logan J, Hitzemannn R, Gatley SJ et al (1996). Cocaine uptake is decreased in the brain of detoxified cocaine abusers. Neuropsychopharmacology 14: $159-168$

Weiss F (2005). Neurobiology of craving, conditioned reward and relapse. Curr Opin Pharmacol 5: 9-19.

Weiss F, Markou A, Lorang MT, Koob GF (1992). Basal extracellular dopamine levels in the nucleus accumbens are decreased during cocaine withdrawal after unlimited-access selfadministration. Brain Res 593: 314-318. 